\title{
Recent perspectives of pediatric mitochondrial diseases (Review)
}

\author{
JUNHUA CAO, HONGWEI WU and ZHENGUANG LI \\ Department of Neonatology, Xuzhou Children's Hospital, Xuzhou, Jiangsu 221002, P.R. China
}

Received May 24, 2017; Accepted September 21, 2017

DOI: $10.3892 /$ etm.2017.5385

\begin{abstract}
Mitochondrial disorders are amongst the most common groups of inborn errors of metabolism. They are caused by deficiencies in the final pathway of the cellular energy production, the mitochondrial respiratory chain. The disorders are clinically and genetically heterogeneous and the aetiology could be found in the mitochondrial, or in the nuclear genome. We searched important e-databases for the collection of latest literature on the mitochondrial disease especially in pediatric population. Most of the studies in the recent past have focused on the understanding of the clinical phenotypes and pathophysiological mechanisms. Leigh syndrome is a common severe, neurodegenerative disease of early childhood. A defect in the POLG gene is another common observation in most of the cases leading to Alpers syndrome. The review concludes that pediatric mitochondrial disorders are severe, progressive and usually multi-systemic. Further, whole genome sequencing
\end{abstract} is an excellent diagnostic option.

16. Prenatal diagnostics

17. Conclusions

\section{Introduction}

Mitochondrial disorders are highly heterogeneous with regard to the clinical phenotype, as well as the genotype (1). The clinical spectrum is extremely broad, from multi-organ, life-threatening disease at birth to single symptoms with onset in middle age. The genetic cause of a mitochondrial disease can be found either in the mitochondrial or in the nuclear genome. We expect approximately one third of the paediatric patients to have disease-causing variants in mitochondrial DNA (mtDNA) (2) and the rest to carry pathogenic variants in nuclear genes. To date, more than 250 nuclear genes have been linked to mitochondrial disease (3). Once a mitochondrial disorder is suspected, the diagnostic procedure is a challenge. There is no specific test to exclude or confirm the diagnosis. Nevertheless, it is of great importance for these patients and their families to establish a definite diagnosis on the genetic level.

\section{Clinical features of mitochondrial disease}

Mitochondrial disorders are clinically heterogeneous. Symptoms can emerge from any organ or tissue, although the central nervous system (CNS) and skeletal muscles are the above all most frequently affected tissues, owing to their high energy demands. In infancy and early childhood, the disease is often multi-systemic, with involvement of not only the CNS and muscles, but also the liver, heart, kidneys and bone marrow, to mention the most frequently involved organs. Early onset of the disease indicates a severe defect in the mitochondrial respiratory chain, and this is related to a poorer prognosis (4). Mitochondrial disorders, with an onset in the adolescence or adulthood, are more often single-organ diseases, such as CPEO, or LHON.

\section{Symptoms and signs from the CNS}

Correspondence to: Dr Zhenguang Li, Department of Neonatology, Xuzhou Children's Hospital, 18 Sudibei Road, Xuzhou, Jiangsu 221002, P.R. China

E-mail: zhenguangli29@163.com

Key words: mitochondrial disorders, pediatrics
Symptoms from the CNS are seen in the majority of children with mitochondrial diseases. The most frequent symptom is a developmental delay (5), which is usually global and affects cognitive, language and motor skills. The end-point cognitive level varies, from mild learning disabilities to severe mental retardation. There is probably no specific cognitive profile since mitochondrial disorders are, in all aspects, extremely 
heterogeneous. A study by Turconi et al indicated a greater impairment in the non-verbal area, particularly the visuospatial abilities. Impairment of the verbal short-term memory (working memory) was also seen (6). Symptoms from the autism spectrum are seen as well, and one hypothesis is that mitochondrial dysfunction can be part of the disease mechanism in autism spectrum disorders in general (7).

Seizures are a frequent complication of mitochondrial disease at all ages. The exact prevalence is not known, but it is estimated to be approximately $40 \%$ (8). Recurrent status epilepticus was seen in as many as $60 \%$ of the patients. Also epileptic syndromes, such as West syndrome and LennoxGastaut syndrome have been reported (9). Alpers syndrome (AS), due to recessive mutations in the POLG gene, is one of the most common mitochondrial syndromes associated with epilepsy (10). AS patients often show focal, myoclonic or complex seizures. Status epilepticus is common, sometimes starting with epilepsia partialis continua, followed by a generalised, therapy-resistant status. Electroencephalography (EEG) may initially show characteristic unilateral, occipital, high-amplitude, slow waves with superimposed polyspikes, evolving into a generalised pattern (11). The energy failure is an important factor, but other aspects of a mitochondrial dysfunction, such as ROS production, disturbed calcium homeostasis and apoptosis are likely to contribute (12). Movement disorders are seen in a substantial proportion of the patients with mitochondrial diseases. In the paediatric population, dystoniasare the most frequent symptoms, and are seen particularly in Leigh syndrome (LS) (13). This is not surprising, as the syndrome includes lesions in the basal ganglia and other extrapyramidal structures, from which these types of symptoms arise. Ataxia is not classified as a movement disorder, but it is a common symptom in several mitochondrial phenotypes caused by mutations in either mtDNA or nDNA (14). Neurological symptom in mitochondrial disease are often progressive, and sometimes rapid, with developmental arrest and loss of skills. The progression can also be stepwise, with a preceding infection or other catabolic situation. Some patients have a very slow progression, appearing like a static condition. A considerable proportion of patients suffer from acute neurological events, such as strokelike episodes, status epilepticus, coma, vomiting or lethargy.

\section{Symptoms from skeletal muscle}

Myopathy is the above all most common single symptom in mitochondrial disease. It is often part of an encephalomyopathy with additional symptoms from other organs, but pure myopathic presentations are seen in the adult, as well as the paediatric population. The isolated mitochondrial myopathy typically presents with axial and proximal muscle weakness. Distal weakness has been reported in sporadic cases in the myopathic group and occurs regularly in the group of patients with mitochondrial polyneuropathies and neurogenic muscle weakness (15). Exercise intolerance and a general fatigue are other hallmarks of the mitochondrial myopathy. Infantile-onset mitochondrial myopathies are usually severe disorders with pronounced weakness, hypotonia and a need for ventilation support and intensive care. It is important to be aware of a subset of patients with this severe phenotype and a cytochrome $c$ oxidase (COX)-deficiency, who turn out to have a reversible disease.

\section{Ophthalmological manifestations}

Ophthalmological findings in mitochondrial disease are common, although the frequency remains unclear (16). The extraocular muscles are strongly dependent on a sufficient energy supply, with mitochondria occupying approximately $60 \%$ of the cell volume (17). It is therefore not surprising, that external ophthalmoplegia is a common finding in patients with mitochondrial disorders. Optic atrophy is often part of a systemic disease with CNS involvement, as in LS. It can also appear in isolation, such as in patients with LHON. In this disease, the function of the retinal ganglion cells is specifically affected, which results in subacute, painless, bilateral visual failure (18). Occasionally, additional symptoms could be seen, preferentially from the nervous system (19). The onset of disease usually occurs in young adulthood, but childhood onset is also seen. Pigmentary retinopathy is another non-specific sign of retinal dysfunction that has been associated with a variety of mtDNA and nDNA mutations. Other abnormalities of the eye and/or vision to be mentioned are cataract, cortical blindness and homonymous hemianopsy.

\section{Hepatopathy and gastrointestinal symptoms}

Hepatic disease is estimated to occur in $10-20 \%$ of patients with mitochondrial disease and usually presents in early childhood $(20)$. The spectrum of severity ranges from transient elevated liver transaminases to acute, fatal liver failure early in life. The eauses of mitochondrial hepatopathies are mainly: i) Disorders of mtDNA maintenance, ii) defects in mitochondrial protein synthesis, iii) defects of RC complex assembly and iv) disorders of the mitochondrial lipid membranes (21).

Gastrointestinal symptoms are common in mitochondrial disorders, regardless of the genetic backgrounds, although they are more prominent in association with certain defects. The mechanism behind the symptoms varies and is sometimes caused by a combination of different tissue/organ involvements. Mitochondrial neuro-gastrointestinal encephalomyopathy (MNGIE), caused by a deficiency of thymidine phosphorylase, due to mutations in TYMP, is characterized by severe gastrointestinal dysmotility, and even a chronic intestinal pseudoobstruction (22). The syndrome frequently presents in adolescence or young adulthood and additional features are cachexia, peripheral neuropathy and/or ophthalmoplegia. Hearing impairment is common and most patients develop a leukoencephalopathy in adulthood (23). Similar phenotypes, with severe gastrointestinal dysmotility, have been reported with mutations in other genes involved in mtDNA maintenance, POLG being one example (24).

Diarrhoea, owing to exocrine pancreas insufficiency, is a cardinal feature of Pearson syndrome. Pearson syndrome is the most frequently seen phenotype in the early onset of a disease caused by a large-scale deletion in mtDNA. Additional symptoms in Pearson syndrome are transfusion-dependent anaemia and lactic acidosis (25). Liver failure, renal tubular acidosis and diabetes mellitus can further complicate the clinical picture. Patients with LS, described in detail below, often have more diffuse gastrointestinal symptoms, such as failure to thrive, feeding difficulties and vomiting. The causative factors behind these symptoms are probably multiple 
in nature, including involvement of the CNS, gastrointestinal tract, muscles and peripheral nerves.

\section{Endocrine dysfunction}

Steroid hormones are synthesized within the mitochondria and a dysfunction of ATP production leads to impaired hormone production and endocrinological symptoms. Overall, endocrinological manifestations seem to be most common in the phenotypes caused by defects of mtDNA, particularly large-scale deletions and point mutations in tRNA genes. Patients with nuclear gene defects may also present with these symptoms, most frequently involving gene defects affecting mtDNA maintenance and translation (26).

Diabetes mellitus is the best described endocrine manifestation. The mechanism of diabetes in mitochondrial disease is not only a matter of decreased insulin secretion owing to a deficient ATP supply, but is also caused by the impairment of the mitochondrial role as a glucose sensor, connecting glucose metabolism to insulin release (27). Diabetes mellitus is also frequently seen in Kearns-Sayre syndrome, caused by large-scale deletions in mtDNA. In Pearson syndrome, exocrine pancreas dysfunction is a more prominent feature, but diabetes is seen as well (28). Short stature is common in patients with mitochondrial disorders. In some of these patients a growth hormone deficiency can be established. In other patients, the underlying mechanisms are yet unknown. Additional endocrinological manifestations that should be mentioned are hypothyroidism, hypoparathyroidism, adrenal insufficiency and hypogonadism.

\section{Symptoms from organ or tissue}

Kidney. Renal manifestations of mitochondrial disease have been reported in association with mtDNA mutations, as wel as numerous nuclear genes. Most usual is a tubular dysfunction, varying from a mild hyperaminoaciduria, which might only occur during illness. The more pronounced tubulopathies are frequently associated with large-scale deletions in mtDNA and the clinical features of Pearson or Keams-Sayre syndrome (29).

Heart. Cardiomyopathies are the most frequent cardiac manifestations of mitochondrial disease and are estimated to occur in 20-40\% of the patients. Hypertrophic cardiomyopathies are most common, but dilated, restrictive and other types are also seen. The severity ranges from asymptomatic, sometimes spontaneously reversible conditions, to a severe cardiomyopathy with an early, even prenatal, onset that causes death in early infancy. The presence of a cardiomyopathy in a mitochondrial disorder, regardless of its severity, is associated with a poorer prognosis (30). Arrythmias, conduction defects and pulmonary hypertension are examples of other more rare cardiac manifestations (31).

Hearing. Hearing impairment/deafness is a symptom of several mitochondrial phenotypes, caused by mutations in mtDNA, as well as in nDNA. The prevalence varies in different studies, but a minimal frequency is approximately $20 \%$ (32).

\section{Leigh syndrome}

$\mathrm{LS}$, is a progressive neurodegenerative disorder of infancy and early childhood. It is the most common pediatric mitochondrial syndrome. It is now confirmed that LS is primarily a disease of the deep grey matter and sometimes involving the white matter. Lesions are characteristically seen in the basal ganglia, thalami, brainstem, cerebellum and spinal cord and consist of areas of demyelination, gliosis, necrosis, spongiosis and vascular proliferation (33). The neurological symptoms include developmental delay/arrest, followed by loss of skills, axial hypotonia, increasing tonus in the arms and legs, ataxia and dystonia. Ophthalmological abnormalities, such as nystagmus and optic atrophy, are frequently seen (34), as well as sensorineural hearing impairment and epilepsy. Additionally, a diversity of non-neurological symptoms, such as cardiomyopathy, hepatopathy, renal tubular dysfunction or hormonal deficiencies, may constitute parts of the phenotype.

LS is most usually caused by a dysfunction of the mitochondrial respiratory chain, although the syndrome can be seen in other inborn errors of metabolism. It is a common phenotype in different conditions that causes severe failure of oxidative metabolism in the mitochondria of the developing brain. The underlying genetic causes are heterogeneous. More than 75 different nuclear genes are reported to be causative (35).

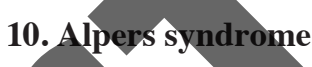

also named Alpers-Huttenlocher syndrome, is another degenerative mitochondrial encephalopathy of infancy and early childhood (36). The phenotype is characterized by intractable epilepsy, developmental regression and hepatopathy with or without liver failure. The disease primarily affects grey matter in the brain, particularly the cerebral cortex, cerebellum and thalami. Pathology in the brain ncludes spongiosis, astrocytosis and neuronal loss. In the liver, hepatitis with fatty degeneration, hepatocyte loss, bile duct proliferation and fibrous scarring, with or without cirrhosis, have been described. In approximately $50 \%$ of the patients, the liver involvement is associated with exposure to sodium valproate (37). Additional symptoms include hypotonia, ataxia and cortical blindness. It is also associated with recessive mutations in the POLG gene (38).

\section{Diagnosing mitochondrial disease}

A detailed medical and family history and a thorough examination are essential for the further diagnostics. The family history may help to discriminate between maternal (indicating an mtDNA defect) and Mendelian inheritance of the disease. Clinical investigations include neurological, cardiac and ophthalmological evaluations and assessments of hearing, growth and psycho-motor development. The finding of multiple organ involvement, especially the brain and muscles, further strengthens the suspicion of a mitochondrial disorder. The mapping of clinical symptoms and signs also serves to establish the extent of disease in order to plan the management and follow-up of the specific individual.

Many of the more frequent symptoms, such as developmental retardation, hypotonia and failure to thrive, are non-specific and seldom raise the suspicion of a mitochondrial disorder. Other symptoms, or constellations of symptoms, are less frequent and more specific and point directly to the mitochondria. Ataxia, external ophthalmoplegia and renal 
tubulopathy are examples of 'red flags', signalling a potential mitochondrial disorder (39). Certain constellations of symptoms may even be clues to a specific mitochondrial syndrome, such as a combination of stroke-like episodes, diabetes and hearing impairment, strongly suggesting the MELAS syndrome.

\section{Neuroimaging}

Neuroimaging is important in all patients with CNS involvement. Structural MRI is the standard investigation. Modern functional brain imaging methods, such as magnetic resonance spectroscopy, diffusion weighted imaging and perfusion MRI may provide valuable information regarding brain metabolism (39). Non-specific findings of cerebral atrophy or leukodystrophy are common. Certain imaging patterns are more distinct, and may be helpful in further biochemical and genetic investigations, such as identification of typical features of LS or AS. Stroke-like lesions, predominantly located in grey matter and not following vascular territories, suggest a MELAS syndrome (40).

\section{Clinical chemistry}

Routine parameters, such as a full blood count, glucose, creatine kinase (CK) and laboratory parameters of liver, parathyroid, thyroid and renal function are evaluated to characterise the systemic involvement of the disease.

Lactic acid is an important, although non-specific biomarker of mitochondrial disease. A substantial propor tion of patients may have consistently normal, or minimally elevated, lactate levels in the blood, as well as the CSF (39) Conversely, elevated blood or CSF lactate levels are seen in a range of conditions not linked to RC disorders. Inappropriate collection or handling of the samples may also result in a high concentration of lactate in the samp

The more specific metabolic work-up serves to exclude other metabolic differentialdiagnoses and to find abnormalities, which further strengthen the suspicion of a mitochondrial disorder. Urinary organic acids are included in the diagnostics of virtually alt types of metabolic disorders. Patients with mitochondrial disorders might have normal excretion, although abnormalities frequently occur. Most common is a non-specific increased excretion of lactate. Acylcarnitine profiles may reveal primary organic acidaemias, primary fatty acid oxidation disorders on a secondary fatty acid oxidation dysfunction due to the OXPHOS defect (42).

\section{Muscle biopsy}

Muscle biopsy is the golden standard procedure in investigations of mitochondrial function. Muscle morphology is studied by means of light and electron microscopy, using histochemical and immunohistochemical methods (43). The finding of 'ragged red fibres' (RRF) is strongly suggestive of a mitochondrial disorder. RRF is a pattern caused by subsarcolemmal accumulation of mitochondria. The presence of fibres deficient in COX activity is another hallmark of mitochondrial disease. Neither RRF nor COX-negative fibres are specific for a mitochondrial disorder, but they may appear secondarily to other, non-mitochondrial myopathies (44). Electron micros- copy may demonstrate a variety of abnormalities associated with mitochondrial disease. The mitochondria often observed to be enlarged, with abnormal shapes, absent cristae and paracrystalline inclusions.

\section{Molecular genetics}

Further genetic tests are performed based on the findings in the muscle biopsy $(45,46)$. Isolated enzyme complex deficiencies indicate mutations in genes encoding subunits of the complex or assembly factors. Complexes I, III, IV and V contain subunits encoded by mtDNA as well as nDNA, whereas a complex II deficiency is expected to be caused by mutations in nuclear genes. Combined enzyme deficiencies including complexes I, III, IV and V indicate a deficiency of mtDNA expression or maintenance. Causative mutations may be found in mitochondrial tRNA genes, but more often in nuclear genes. Occasionally, genetic analyses are performed without a preceding mitochondrial assay in muscle tissue $\mathrm{LHON}$ is an example of that, with a specific clinical picture which, in more than $90 \%$ of the patients, is caused by one of three different mtDNA mutations.

Sequence analysis of the entire mitochondrial genome is usually the first step in the molecular part of the diagnostic procedure. It is relatively easily done and, if negative, rules out maternal (mitochondrial) inheritance of the disease. mtDNA mutation analyses are preferably performed in muscle tissue, since mtDNA molecules harbouring point mutations or large deletions tend to accumulate in non-dividing cells, such as muscle and nerve cells, but are eliminated in the rapidly dividing blood cells (47). Urinary epithelial cells and buccal mucosa cells are alternative cell types, with the advantage of a non-invasive sample collection. Once a causative mutation in $m \mathrm{DNA}$ is excluded, hundreds of nuclear genes remain to be investigated. Sanger sequence analyses of selected genes are seldom cost-effective since identical signs and symptoms may be caused by mutations in many different genes.

The new techniques of massively parallel DNA sequencing have greatly increased our ability to establish a genetic diagnosis in patients with mitochondrial disorders, and have recently been implemented in the clinical setting $(48,49)$. As prices have fallen and techniques have further developed, the use of whole genome sequencing (WGS) is preferred and has gradually been implemented at many centres for mitochondrial diagnostics. WGS enables variant detection also in non-coding regions, such as the introns. A WES analysis identifies approximately 20,000 single nucleotide variants (SNVs) per genome, whereas WGS identifies as many as four million.

\section{Prenatal diagnostics}

Prenatal diagnostic analyses of mitochondrial disease are usually based on genetic findings (50). In families with a disorder caused by identified mutations in a nuclear gene, molecular analyses in a chorionic villus sample (CVS) or cultured amniocytes could be performed in a customary manner. A preimplantatory genetic diagnosis (PGD) might also be an option for these families. Prenatal diagnostics of mtDNA mutations are more complicated owing to the fact that most pathogenic mtDNA mutations are heteroplasmic. Preimplantatory genetic diagnostics is currently used only to a limited extent. 


\section{Conclusions}

It was concluded from the above that pediatric mitochondrial disorders are severe, progressive and usually multi-systemic. Further, WGS is an excellent diagnostic option.

\section{References}

1. Lunsing RJ, Strating K, de Koning TJ and Sijens PE: Diagnostic value of MRS-quantified brain tissue lactate level in identifying children with mitochondrial disorders. Eur Radiol 27: 976-984, 2017.

2. Kirby DM and Thorburn DR: Approaches to finding the molecular basis of mitochondrial oxidative phosphorylation disorders. Twin Res Hum Genet 11: 395-411, 2008.

3. Kohda M, Tokuzawa Y, Kishita Y, Nyuzuki H, Moriyama Y, Mizuno Y, Hirata T, Yatsuka Y, Yamashita-Sugahara Y, Nakachi Y, et al: A comprehensive genomic analysis reveals the genetic landscape of mitochondrial respiratory chain complex deficiencies. PLoS Genet 12: e1005679, 2016.

4. Debray FG, Lambert M, Chevalier I, Robitaille Y, Decarie JC, Shoubridge EA, Robinson BH and Mitchell GA: Long-term outcome and clinical spectrum of 73 pediatric patients with mitochondrial diseases. Pediatrics 119: 722-733, 2007.

5. Pinto M, Pickrell AM and Moraes CT: Regional susceptibilities to mitochondrial dysfunctions in the CNS. Biol Chem 393: 275-281, 2012

6. Turconi AC, Benti R, Castelli E, Pochintesta S, Felisari G, Comi G, Gagliardi C, Del Piccolo L and Bresolin N: Focal cognitive impairment in mitochondrial encephalomyopathies: A neuropsychological and neuroimaging study. J Neurol Sci 170: 57-63, 1999.

7. Palmieri L and Persico AM: Mitochondrial dysfunction in autism spectrum disorders: Cause or effect? Biochim Biophys Acta 1797: 1130-1137, 2010.

8. Rahman S: Mitochondrial disease and epilepsy. Dev Med Neurol 54: 397-406, 2012.

9. Khurana DS, Salganicoff L, Melvin JJ, Hobdell EF, Valencia Hardison HH, Marks HG, Grover WD and Legido A: Epilep. and respiratory chain defects in children with mitochondria encephalopathies. Neuropediatrics 39: 8-13

10. Bindoff LA and Engelsen BA: Mitochond epilepsy. Epilepsia 53 (Suppl 4): 92-97,

11. Wolf NI, Rahman S, Schmitt B Harting I, Wohlrab G, Ebinger F, epilepticus in children with Alpers' disease caused by POIG1 Epilepsia 50: 1596-1607,2009.

12. Rahman S: Pathophysiology of mitochondrial disease causing epilepsy and status epilepticur. Epilepsy Behav 49: 71-75, 2015.

13. Martikainen MH, Ng YS, Gorman GS, Alston CL, Blakely EL, Schaefer AM, Chinnery PF, Burn DJ, Taylor RW, McFarland R and Turnbull DM: Clinical, genetic, and radiological features of extrapyramidal movement disorders in mitochondrial disease. JAMA Neurol 73: 668-674, 2016

14. Lax NZ, Hepplewhite PD, Reeve AK, Nesbitt V, McFarland R, Jaros E, Taylor RW and Turnbull DM: Cerebellar ataxia in patients with mitochondrial DNA disease: A molecular clinicopathological study. I Neuropathol Exp Neurol 71: 148-161, 2012.

15. Giordano C, Pichiorri F, Blakely EL, Perli E, Orlandi M, Gallo P, Taylor RW, Inghilleri M and d'Amati G: Isolated distal myopathy of the upper limbs associated with mitochondrial DNA depletion and polymerase gamma mutations. Arch Neurol 67: 1144-1146, 2010.

16. Zhu CC, Traboulsi EI and Parikh S: Ophthalmological findings in 74 patients with mitochondrial disease. Ophthalmic Genet 38: 67-69, 2017.

17. Grönlund MA, Honarvar AK, Andersson S, Moslemi AR Oldfors A, Holme E, Tulinius M and Darin N: Ophthalmological findings in children and young adults with genetically verified mitochondrial disease. Br J Ophthalmol 94: 121-127, 2010.

18. Leber $\mathrm{T}$ : Ueber hereditäre und congenital-angelegte Sehnervenleiden. Albrecht Von Graefes Arch Ophthalmol 17: 249-291, 1871.

19. Newman NJ: Leber's hereditary optic neuropathy. New genetic considerations. Arch Neurol 50: 540-548, 1993.

20. Cormier-Daire V, Chretien D, Rustin P, Rötig A, Dubuisson C Jacquemin E, Hadchouel M, Bernard O and Munnich A: Neonatal and delayed-onset liver involvement in disorders of oxidative phosphorylation. J Pediatr 130: 817-822, 1997.
21. Rahman S: Gastrointestinal and hepatic manifestations of mitochondrial disorders. J Inherit Metab Dis 36: 659-673, 2013

22. Garone C, Tadesse S and Hirano M: Clinical and genetic spectrum of mitochondrial neurogastrointestinal encephalomyopathy. Brain 134: 3326-3332, 2011.

23. Hirano M, Nishigaki Y and Martí R: Mitochondrial neurogastrointestinal encephalomyopathy (MNGIE): A disease of two genomes. Neurologist 10: 8-17, 2004

24. Spiegler J, Stefanova I, Hellenbroich Y and Sperner J: Bowel obstruction in patients with Alpers-Huttenlocher syndrome. Neuropediatrics 42: 194-196, 2011

25. Crippa BL, Leon E, Calhoun A, Lowichik A, Pasquali M and Longo N: Biochemical abnormalities in Pearson syndrome. Am J Med Genet A 167A: 621-628, 2015

26. Chow J, Rahman J, Achermann JC, Dattani MT and Rahman S: Mitochondrial disease and endocrine dysfunction. Nat Rev Endocrinol 13: 92-104, 2017.

27. Maechler P: Mitochondrial function and insulin secretion. Mol Cell Endocrinol 379: 12-18, 2013

28. Karaa A and Goldstein A:The spectrum of clinical presentation, diagnosis, and management of mitochondrial forms of diabetes. Pediatr Diabetes 16:

29. Martín-Hernández E, García-Silva MT, Vara J, Campos Y, Cabello A, Muley R, Del Hoyo P, Martín MA and Arenas J: Renal pathology in children with mitochondrial diseases. Pediatr Nephrol 20:

30. Holmgren D, Wåhlander H, Eriksson BO, Oldfors A, Holme E and Tulinius $M$ : Cardiomyopathy in ehildren with mitochondrial disease; clinical course and cardiological findings. Eur Heart $\mathrm{J} 24: 280-288,2003$

31. Finsterer J and Kothari S: Cardiac manifestations of primary nitochondrial disorders. Int J Cardiol 177: 754-763, 2014.

Gold M and Rapin I: Non-Mendelian mitochondrial inheritance a cause of progressive genetic sensorineural hearing loss. Int J Pediatr Otorhinolaryngol 30: 91-104, 1994

33. Cavanagh JB and Harding BN: Pathogenic factors underlying Leigh's disease. Tissue responses to cellular energy deprivation and their clinico-pathological consequences. Brain 117: 1357-1376, 1994

rand R, Andersson S, Seyedi Honarvar AK, Sofou K, N, Tulinius M and Grönlund MA: Ophthalmological characteristics in children with Leigh syndrome - A long-term follow-up. Acta Ophthalmol 94: 609-617, 2016.

. Lake NJ, Compton AG, Rahman S and Thorburn DR: Leigh syndrome: One disorder, more than 75 monogenic causes. Ann Neurol 79: 190-203, 2016

36. Khan A, Trevenen C, Wei XC, Sarnat HB, Payne E and Kirton A: Alpers syndrome: The natural history of a case highlighting neuroimaging, neuropathology, and fat metabolism. J Child Neurol 27: 636-640, 2012.

37. Isohanni P, Hakonen AH, Euro L, Paetau I, Linnankivi T, Liukkonen E, Wallden T, Luostarinen L, Valanne L, Paetau A, et al: POLG1 manifestations in childhood. Neurology 76: 811-815, 2011.

38. Anagnostou ME, Ng YS, Taylor RW and McFarland R: Epilepsy due to mutations in the mitochondrial polymerase gamma (POLG) gene: A clinical and molecular genetic review. Epilepsia 57: 1531-1545, 2016.

39. Haas RH, Parikh S, Falk MJ, Saneto RP, Wolf NI, Darin N and Cohen BH: Mitochondrial disease: A practical approach for primary care physicians. Pediatrics 120: 1326-1333, 2007.

40. Dinopoulos A, Cecil KM, Schapiro MB, Papadimitriou A, Hadjigeorgiou GM, Wong B, deGrauw T and Egelhoff JC: Brain MRI and proton MRS findings in infants and children with respiratory chain defects. Neuropediatrics 36: 290-301, 2005.

41. Haas RH, Parikh S, Falk MJ, Saneto RP, Wolf NI, Darin N, Wong LJ, Cohen BH and Naviaux RK; Mitochondrial Medicine Society's Committee on Diagnosis: The in-depth evaluation of suspected mitochondrial disease. Mol Genet Metab 94: 16-37, 2008.

42. Sim KG, Hammond J and Wilcken B: Strategies for the diagnosis of mitochondrial fatty acid beta-oxidation disorders. Clin Chim Acta 323: 37-58, 2002 .

43. Larsson NG and Oldfors A: Mitochondrial myopathies. Acta Physiol Scand 171: 385-393, 2001.

44. Yamamoto M, Koga Y, Ohtaki E and Nonaka I: Focal cytochrome $c$ oxidase deficiency in various neuromuscular diseases. J Neurol Sci 91: 207-213, 1989.

45. Zhang M, Si Y and Zhao J: Characteristics of molecular genetics and research progress on mitochondrial diseases. Zhonghua $\mathrm{Yi}$ Xue Yi Chuan Xue Za Zhi 33: 717-725, 2016 (In Chinese). 
46. Hsu YC, Chen CT and Wei YH: Mitochondrial resetting and metabolic reprogramming in induced pluripotent stem cells and mitochondrial disease modeling. Biochim Biophys Acta 1860: 686-693, 2016.

47. Ferreira A, Serafim TL, Sardão VA and Cunha-Oliveira T: Role of mtDNA-related mitoepigenetic phenomena in cancer. Eur J Clin Invest 45 (Suppl 1): 44-49, 2015.

48. Stranneheim $\mathrm{H}$ and Wedell A: Exome and genome sequencing: A revolution for the discovery and diagnosis of monogenic disorders. J Intern Med 279: 3-15, 2016.

49. Yang Y, Muzny DM, Reid JG, Bainbridge MN, Willis A, Ward PA, Braxton A, Beuten J, Xia F, Niu Z, et al: Clinical whole-exome sequencing for the diagnosis of mendelian disorders. N Engl J Med 369: 1502-1511, 2013.
50. Faivre L, Cormier-Daire V, Chrétien D, Christoph von KleistRetzow J, Amiel J, Dommergues M, Saudubray JM, Dumez Y, Rötig A, Rustin P and Munnich A: Determination of enzyme activities for prenatal diagnosis of respiratory chain deficiency. Prenat Diagn 20: 732-737, 2000.

(c) (i) $(9)$ This work is licensed under a Creative Commons cc) At No No Atribution-NonCommercial-NoDerivatives 4.0 International (CC BY-NC-ND 4.0) License.

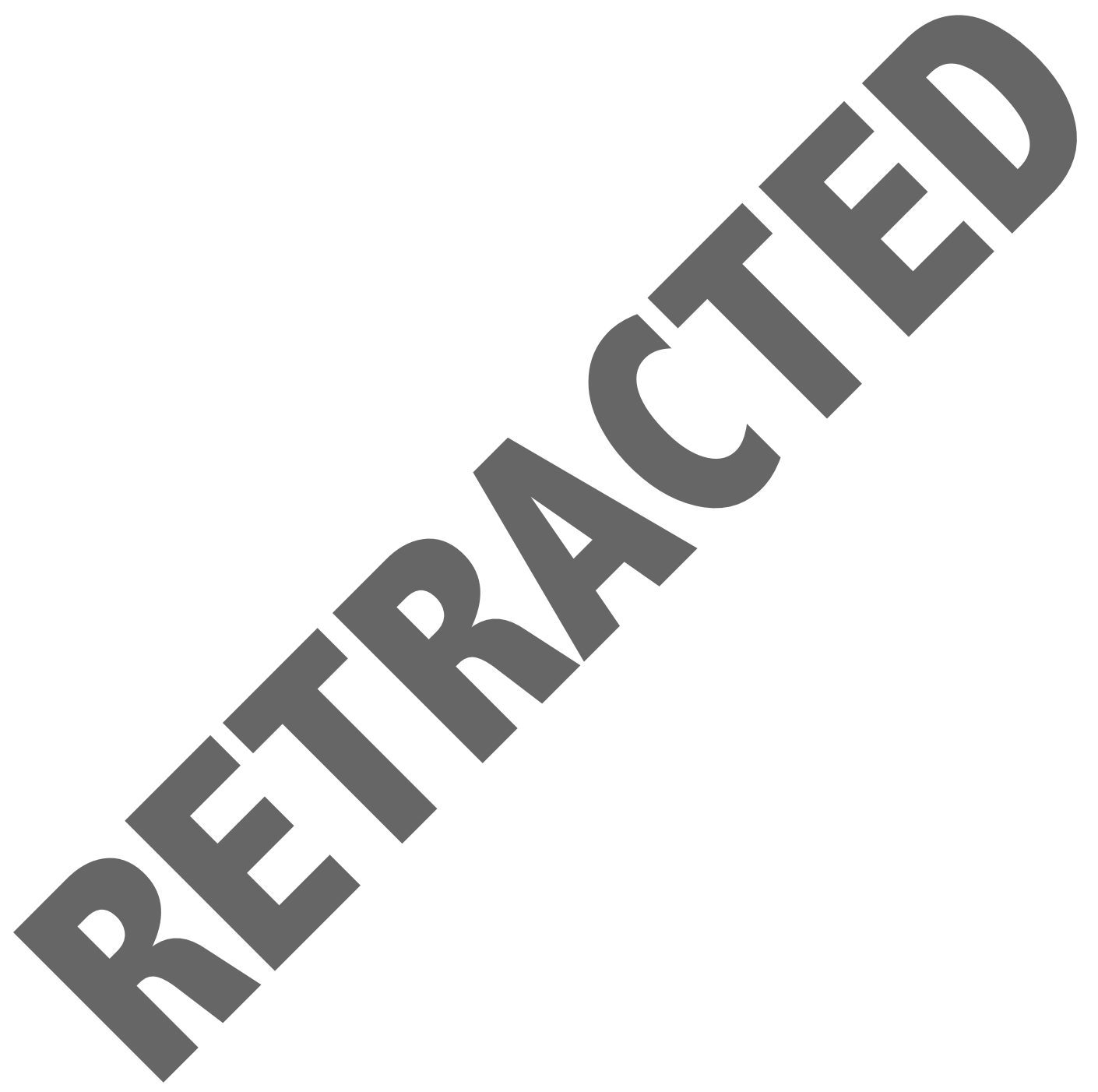

